\title{
Large Tumour Volume Reduction of Idh Mutated Anaplastic Glioma Involving the Insular Region Following Radiation Therapy: an Alternative to Maximal Surgical Resection
}

Gabrielle Metz ( $\sim$ gabrielle.metz@health.nsw.gov.au )

Royal North Shore Hospital

Dasantha Jayamanne

Royal North Shore Hospital

Helen Wheeler

Royal North Shore Hospital

Matthew Wong

Gosford Hospital

Raymond Cook

Royal North Shore Hospital

Nicholas Little

Royal North Shore Hospital

Jonathon Parkinson

Royal North Shore Hospital

Marina Kastelan

Royal North Shore Hospital

Chris Brown

Royal North Shore Hospital

Michael Back

Royal North Shore Hospital

\section{Research Article}

Keywords: anaplastic glioma, IDH mutation, residual volume, radiation therapy

Posted Date: June 18th, 2021

DOl: https://doi.org/10.21203/rs.3.rs-598305/v1

License: (c) (1) This work is licensed under a Creative Commons Attribution 4.0 International License. Read Full License 
Page $2 / 22$ 


\section{Abstract}

\section{Background:}

In IDH-mutated anaplastic glioma (IDHmutAG) of the insular cortex there remains uncertainty of benefit with near-total resection compared to limited surgery with radiation therapy (IMRT). This study aimed to assess tumour volume reduction in patients following IMRT and impact of residual post-surgical volume.

\section{Methods and Materials:}

Patients with IDHmutAG involving insular cortex managed with IMRT from 2008-2019 had baseline patient, tumour and treatment factors recorded. Volumetric assessment of residual disease on MRI was performed at baseline, month +3 and month +12 post-IMRT. Potential prognostic factors were analysed for tumour reduction and relapse-free survival, and assessed by log-rank and Cox regression analyses.

\section{Results:}

32 patients with IDHmutAG of the insular cortex were managed with median follow-up post-IMRT of 67.2 months. Pathology was anaplastic astrocytoma (AAmut) in 20, and anaplastic oligodendroglioma (AOD) in 12 patients. Median pre-IMRT volume on T1 and T2Flair was $24.3 \mathrm{~cm}^{3}$ and $52.2 \mathrm{~cm}^{3}$. Twenty-seven patients were alive with 5 -year relapse-free survival of $80 \%$. There was a median $67 \%$ and $64 \%$ reduction from baseline occurring at 3 months post-IMRT for T1 and T2Flair respectively; and subsequent median $78 \%$ and $73 \%$ at 12 months. At 12 months AOD patients had median $83 \% \mathrm{~T} 1$ volume reduction compared to $63 \%$ in AAmut $(p<0.01)$. There was no difference on T2Flair volume $(p=0.64)$. No other pathological factors influenced volume reduction at 12 months. No factors were associated with relapse-free survival including baseline T1 $(p=0.52)$ and T2Flair $(p=0.93)$ volume.

\section{Conclusion:}

IMRT provides large tumour volume reduction in IDHmutAG of the insular cortex with no significant negative impact of residual disease volume on relapse-free survival.

\section{Introduction}

Anaplastic gliomas (AG) with IDH mutation (IDHmut) involving the insular cortex of the frontal lobe are infiltrative tumours that extend diffusely along neural tract pathways. These tumours occur in younger patients and have median survivals beyond 10-15 years [1-4]. Improved surgical techniques allow an attempt for complete resection $[5,6]$, however due to the anatomical site there is potential risk of long-term post-surgical neurological deficits [7]. Whilst evidence supports an association of complete surgical resection and improved outcome for glioblastoma and IDH wildtype anaplastic glioma [8,9], there is less evidence for similar magnitude of benefit for IDH mutated anaplastic glioma [10]. The landmark data from Berger et al demonstrates an association with surgical volume reduction and outcome, however, this 
was in the era prior to IDH diagnostic classification and additionally $40 \%$ of patients received radiation therapy following surgery [11].

Concurrently improvements in radiation therapy techniques and target volume delineation of tumour can minimise risk of morbidity and potentially provide a greater therapeutic ratio with reduction of risk [12-14]. This study explores the volume reduction, morbidity and survival occurring in patients with AG IDHmut involving the insular cortex managed with IMRT and adjuvant temozolomide.

\section{Methods}

Consecutive adult patients diagnosed with anaplastic glioma and referred to the Neuro-oncology Multidisciplinary Tumour Board at the Northern Sydney Cancer Centre were entered into a prospective Anaplastic Glioma Database, approved by Institutional Ethics Review Board. All methods were performed in accordance with guidelines and regulations as per the Northern Sydney Local Health District Human Research Ethics Committee [15].

\section{Patient selection}

Eligible patients for this study were those patients in the Anaplastic Glioma Database with an IDHmutated tumour with extension into the insular region, and managed with IMRT between January 2008 and June 2019. In the database, histopathological classification was initially recorded as anaplastic astrocytoma (AA), anaplastic oligoastrocytoma (AOA) or anaplastic oligodendroglioma (AOD) based on WHO 2007 Classification [16]. Molecular factors such as 1p19q codeletion, IDH1/2 mutation (evaluated by immunohistochemical and pyrosequencing techniques) and ATRX mutation were recorded where available. The availability of these results varied over the years as new molecular pathology techniques were sequentially introduced into clinical practice [15].

Patients had histopathology subsequently updated as per the WHO 2016 Classification [17]; and included those with recently diagnosed WHO Grade III Pathology or patients with known WHO Grade II Pathology from prior diagnosis but had recent radiological or metabolic progression consistent with anaplastic change. The latter required imaging criteria of progressive MRI T1 radiological abnormality within a sixmonth period, in addition to either new gadolinium enhancement on MRI or FDG uptake in absence of enhancement. These progressive IDH mutated low grade tumours were presumed to have an equivalent natural history to anaplastic glioma [18] and were included in the study.

\section{Radiological Procedures}

Gadolinium-enhanced 3T MRI was the principal diagnostic procedure at initial diagnosis and RT planning. Tumour was delineated on T1 and T2Flair sequences. Presence of contrast enhancement was recorded as absent, patchy $(<10 \mathrm{~mm})$ or diffuse regions $(>10 \mathrm{~mm})[15]$.

Nuclear medicine imaging with combined FET and FDG-PET was commenced in 2011 and subsequently performed routinely on all patients with IDH mutation. The FET studies were acquired on a Siemens 
Biograph mCT PET/CT scanner with extended axial field of view and time of flight (ToF) imaging capability; with 20 minute dynamic and 10 minute static acquisitions following a 3 minute FET infusion period.

\section{$\underline{\text { Radiological Classification }}$}

An insular tumour for this study was defined as having MRI evidence of infiltration into the gyri of the insular cortex. The insular tumours were then classified radiologically as per the Berger-Sanai Classification [19] with involvement of regions classifying the tumours as occupying Zones I-IV with a further category (Zone V) classifying giant tumours that occupy all zones. This classification reflects the geographical site of tumour within the insular region, related superior and inferior to the line of the Sylvian fissure; and anterior/posterior to a perpendicular plane crossing the foramen of Monro. Patients were described to the zones involved as well as the major zone involved.

\section{$\underline{\text { Treatment Procedures }}$}

\section{Neurosurgical Management}

Patients were referred from a number of neurosurgical centres with varying approaches to the extent of surgical intervention and timing of IMRT for anaplastic glioma. Data was recorded regarding the number of salvage craniotomies, the time from initial surgery to radiation therapy and the extent of gross residual disease on MRI T1 and T2Flair sequences prior to IMRT [15].

\section{Radiation Therapy Management}

IMRT was utilised for all patients and management was through one clinician. The target volume defined by preoperative and postoperative MRI and importantly any historical MRI imaging, especially for patients with progressive disease after prior surveillance was imported for assistance with target volume determination. FDG-PET and FET-PET were utilised where available for target volume determination [15].

Prior to 2011, the tumour was defined as a gross tumour volume (GTV) for each imaging modality and separate T1, T2Flair, FDG-PET and FET-PET GTVs were delineated. These were combined to produce a final GTV, expanded by $10 \mathrm{~mm}$ to a clinical target volume (CTV) and then a further $3 \mathrm{~mm}$ to a planning target volume (PTV). This volume received a dose of $59.4 \mathrm{~Gy}$ in 33 fractions and delivered over a period of 6-7 weeks.

From 2011 with the commencement of routine IDH mutation testing and the recognition of a potential favourable subgroup of patients with long-term survival, a new protocol was commenced for patients with IDH mutation [12]. This incorporated an IMRT integrated boost with two dose levels 59.4Gy and 54Gy defined both by MRI and PET with FDG or FET tracers. The high dose region (GTV59.4) encompassed any areas of gadolinium enhancement, T1 density or FDG uptake. The lower dose region (GTV54) included this volume, as well as region of T2Flair and FET uptake outside of this volume. 
Patients with wild-type IDH tumours on both immunohistochemistry and subsequent protein sequencing were managed with a single-phase treatment to $59.4 \mathrm{~Gy}$ as per the EORTC CATNON Protocol $[15,20]$.

\section{Systemic Therapy Management}

The protocol for systemic therapy evolved over the duration of study with the addition of adjuvant temozolomide commencing in early 2012 following release of long-term results of the RTOG and EORTC studies showing improved median survival for patients with oligodendroglial tumours receiving adjuvant chemotherapy $[21,22]$. This was extrapolated to the cohort of patients with IDH mutation rather than just those oligodendroglial tumours with 1p19q co-deletion [15].

\section{Post-treatment Surveillance}

Patients were evaluated at one month post-IMRT with MRI and then commenced a surveillance programme with 3 monthly MRI for years 1-2, then 4 monthly in year 3 followed by 6 monthly until year 5 [15].

\section{Study Procedures}

\section{MRI Tumour Volume Calculation}

Measurements were performed by the lead author, an advanced trainee radiation oncologist. The volume of residual tumour pre-RT was calculated in $\mathrm{cm}^{3}$ by contouring the complete volume on MRI fused to the simulation CT scan in the treatment planning system. Surgical cavity was excluded in selection of the maximum dimension in any plane. This was conducted on T1 and T2Flair image sequences (Figure 1). Any uncertainty over volume delineation was clarified with a neuroradiologist [15].

At set time points for study evaluation, three months (month+3) and twelve months (month +12$)$ following completion of RT, the same procedure was undertaken on these images fused into the radiation therapy treatment planning system (Figure 2).

Additionally, the presence of contrast enhancement was recorded from T1 with gadolinium sequences and categorised as absent, patchy $(<10 \mathrm{~mm})$ or diffuse regions $(>10 \mathrm{~mm})$. Where measurable, the volume of the largest contrast enhancing area was calculated at each time point.

\section{Study Endpoints}

The primary endpoint was change in the MRI tumour volume as measured on both T1 and T2Flair sequences performed at 12 months post-IMRT completion. A secondary endpoint was the similar procedure at 3 months post-RT completion. The volumetric endpoint was calculated as a median reduction for the total group; as well as the median of the volume reductions experienced by each patient.

Relapse-free survival (as measured from date of commencement of RT to date of relapse or last followup) was analysed as a measure of treatment efficacy. Relapse was defined using RANO Criteria post 
2012 [23]. For patients within 18 months of completion of IMRT, in which uncertainty existed whether the MRI changes were related to tumour or treatment effect, sequential MRIs were utilised to confirm diagnosis but date of relapse was taken from initial MRI if subsequently confirmed. The relapse site was categorised in relation to the IMRT defined GTV (CTV59.4) and defined as: Infield (>50\% within CTV59.4/54); Marginal ( $>50 \%$ within $20 \mathrm{~mm}$ of CTV59.4/54); Distant ( $>50 \%$ beyond $20 \mathrm{~mm}$ from CTV59.4/54); or Combined (synchronous sites of relapse). Additionally, involvement of ventricular system was reported if a distant relapse was evident.

No detailed acute or late toxicity criteria were assessed for this study; however a measurement of longer performance status and impact of therapy were explored using functional outcome measures obtained at month +12 and month +60 post-IMRT. These were change in Eastern Co-operative Oncology Group Scale (ECOG) as well as change in Employment Status from baseline.

\section{$\underline{\text { Statistical Considerations }}$}

All patients had clinical and MRI volume data entered on an Excel database at Northern Sydney Cancer Centre and updated for outcome events. Kaplan-Meier estimates of survival distribution were used to calculate relapse-free survival. Volume reductions were calculated at each time point from baseline as both the central tendency measured as median score; and the dispersion of data by the interquartile range (q1-3). Log-rank test was used to investigate associations between the volume reduction and potential predictive factors. All reported p-values are two-tailed. Statistical significance was defined as $p<0.05$ in all cases. IBM SPSS Statistics Version 23 was used for statistical analysis [15].

\section{Results}

One hundred and sixty-one patients were accessed from the Anaplastic Glioma Database who had IDH mutated anaplastic glioma and received management between January 2008 and June 2019. Thirty-two patients had tumours infiltrating into the insular region and were evaluable for the study. At time of data censure in February 2020, five patients are deceased with a 5-year overall survival of 92\% (Cl 0.81-1.00) (Fig. 3). The median follow-up for survivors is 67.2 months with a range from 6 months to 134.4 months.

Patient characteristics are listed in Table 1. Median age was $38.3 \mathrm{yrs}$. Forty-seven percent of patients were managed with IMRT at initial diagnosis; and $16 \%$ of patients at second or later relapse. 
Table 1

Patient, Tumour and Treatment Characteristics

\begin{tabular}{|c|c|c|}
\hline & Subgroup & $\begin{array}{l}\text { Number }(n=32) \\
(\%)\end{array}$ \\
\hline \multirow[t]{3}{*}{ Age at Diagnosis } & $<40$ yrs & 17 \\
\hline & $>40 \mathrm{yrs}$ & 15 \\
\hline & Median & 38 yrs \\
\hline \multirow[t]{3}{*}{ Number of Craniotomy prior to RT } & 1 & $23(72 \%)$ \\
\hline & 2 & $4(12 \%)$ \\
\hline & 3 or more & $5(16 \%)$ \\
\hline \multirow[t]{4}{*}{ Timing of RT } & At initial diagnosis & $15(47 \%)$ \\
\hline & 1st relapse & $12(38 \%)$ \\
\hline & 2nd relapse & $2(6 \%)$ \\
\hline & Later & $3(9 \%)$ \\
\hline Pathology & AOD & $12(37 \%)$ \\
\hline WHO 2016 & AAmut & $20(63 \%)$ \\
\hline Ki67\% (preIMRT Surgery) & Median $(n=22)$ & $10.0 \%$ (q1-3: 5.0-16.2) \\
\hline Ki67\% (prior Surgery) & Median $(n=10)$ & $5.0 \%$ (q1-3: 2.5-6.5) \\
\hline \multirow[t]{3}{*}{ ATRX } & Retained & $16(50 \%)$ \\
\hline & Lost & $8(25 \%)$ \\
\hline & Unknown & $8(25 \%)$ \\
\hline Pre-Treatment & Nil & $27(84 \%)$ \\
\hline \multirow[t]{2}{*}{ Gad enhancement } & $<10 \mathrm{~mm}$ & $4(12 \%)$ \\
\hline & $>10 \mathrm{~mm} /$ diffuse & $1(3 \%)$ \\
\hline Pre IMRT ECOG & 0 & $13(41 \%)$ \\
\hline \multirow[t]{3}{*}{ Performance Status } & 1 & $17(53 \%)$ \\
\hline & 2 & $1(3 \%)$ \\
\hline & 3 & $1(3 \%)$ \\
\hline \multirow[t]{2}{*}{ IMRT Technique } & Integrated Boost & 27 (84\%) \\
\hline & Single & $5(16 \%)$ \\
\hline
\end{tabular}




\begin{tabular}{|lll|}
\hline & Subgroup & $\begin{array}{l}\text { Number }(\mathbf{n = 3 2}) \\
(\%)\end{array}$ \\
\hline Temozolomide & Nil & $7(22 \%)$ \\
& Pre-and post-RT & $2(6 \%)$ \\
& Adjuvant post-RT & $23(72 \%)$ \\
\hline
\end{tabular}

All patients had tumours with IDH mutation, and pathologies as per WHO 2016 Classification were AOD in 12 patients and AAmut in 20 patients. IMRT was given immediately after the index surgery in 22 patients, whilst 10 of the 32 patients were managed following progression from a prior known anaplastic (4 patients) or low-grade glioma ( 6 patients). Of the 22 patients with surgery immediately before IMRT the median ki67\% was 10\% (q1-3: 5.0 -16.2); whilst the 10 patients with no recent surgery the median ki67\% was $5 \%$ (q1-3: 2.5-6.5).

All patients had radiologically-measurable residual disease on MRI/FET-PET evident prior to IMRT.

Twenty-one patients had a maximum diameter of residual tumour exceeding 50mm; and 16 patients had gadolinium enhancement prior to diagnosis, though only 5 had residual enhancement at time of IMRT. The bulk of disease is reflected by the Berger-Sanai Classification demonstrating $53 \%$ of patients had a giant glioma with involvement of all 4 zones. Zone I (anterior and superior) was involved in $84 \%$; though it was only the dominant region in $37 \%$ of patients.

All 32 patients received the IMRT to a dose of 59.4Gy as prescribed, and 27 had the integrated boost technique. Sequential temozolomide chemotherapy was delivered in $78 \%$ of patients; with 7 patients managed with RT alone prior to the protocol alteration in 2012 when sequential temozolomide was added routinely.

MRI Tumour Volume Analysis

At baseline the 32 patients had median T1 and T2Flair volumes of $24.3 \mathrm{~cm}^{3}$ (range: $4.6-107.0$ ) and 52.2 $\mathrm{cm}^{3}$ (range: 5.0-202.0) respectively (Table 2). The 5 patients with contrast enhancement at baseline had minimal enhancement with one patient having $4 \mathrm{~cm}^{3}$ but the other four patients were patchy enhancement and less than $0.1 \mathrm{~cm}^{3}$. Following treatment, all 32 patients were assessable at month +3 and 30 patients had month +12 assessment. 
Table 2

Baseline Tumour Volume Parameters pre-IMRT

\begin{tabular}{lll|}
\hline Endpoint & $\mathbf{n = 3 2}(\%)$ \\
\hline 2D Residual Tumour & $<20 \mathrm{~mm}$ & $2(6 \%)$ \\
(Maximum diameter) & $20-50 \mathrm{~mm}$ & $9(28 \%)$ \\
& $>50 \mathrm{~mm}$ & $21(66 \%)$ \\
T1 Volume & $24.3 \mathrm{~cm}^{3}$ & $4.6-107.0 \mathrm{~cm}^{3}$ \\
(Median and range) & & \\
T2 Volume & $52.2 \mathrm{~cm}^{3}$ & $5.0-201.9 \mathrm{~cm}^{3}$ \\
(Median and range) & & \\
\hline Berger-Sanai Classification Zones & I & $27(84 \%)$ \\
(Number and \% tumours where Zone involved) & II & $20(62 \%)$ \\
& III & $18(56 \%)$ \\
& IV & $26(81 \%)$ \\
& Giant & $17(53 \%)$ \\
\hline Principal Berger-Sanai Classification Zone & I & $12(38 \%)$ \\
(Major Zone Involved) & II & $0(0 \%)$ \\
& III & $3(9 \%)$ \\
& IV & $9(25 \%)$ \\
& Giant & $9(28 \%)$ \\
\hline
\end{tabular}

Fig, 4 Individual patient absolute MRI Tumour Volume (T1 and T2Flair) reductions at month +3 and month +12 post-IMRT for pathological subtype.

The individual patients' volume reduction and the rate of change for both T1 and T2Flair volumes at month +3 and month +12 are demonstrated in Fig. 4. The median T1 volume reduced from $24.3 \mathrm{~cm}^{3}$ to $6.3 \mathrm{~cm}^{3}$ and $6.2 \mathrm{~cm}^{3}$ at months +3 and +12 respectively. The median T2Flair volumes paralleled this reduction from $52.2 \mathrm{~cm}^{3}$ to $16.1 \mathrm{~cm}^{3}$ and $12.4 \mathrm{~cm}^{3}$ respectively. At month +3 , the median volume reduction for T1 and T2Flair was by $67 \%$ and $64 \%$ respectively; which continued to reduce to $78 \%$ and $73 \%$ from baseline at month +12 .

For individual patients and pathological subtype, the volume reduction at month +12 are shown as Waterfall Plots (Fig. 5). At month $+12,56 \%$ and $44 \%$ of patients had $>75 \%$ volume reduction in $\mathrm{T} 1$ and T2Flair volume from baseline. 
For the 5 patients with contrast enhancement prior to IMRT, the volume measurements were limited with no consistent pattern of response. At month $+3,2$ patients had resolution of their enhancement, but a further 1 patient had developed focal enhancement of less than $2.3 \mathrm{~cm}^{3}$. The one patient with prior bulky enhancement of $4 \mathrm{~cm}^{3}$ had a complete response by month +3 .

Predictive factors for MRI Tumour Volume reduction at month +12

Tumour related factors were analysed for association with $\mathrm{T} 1$ and T2Flair tumour volume reduction at month +12 . Only WHO 2016 pathological subtype of AOD compared with AAmut was associated with more $\mathrm{T} 1$ volume reduction with median $83 \%$ and $63 \%$ respectively $(p<0.01)$. This difference was not evident with T2Flair reduction at month +12 with median $76 \%$ and $71 \%$ respectively $(p=0.62)$. No other factors were associated with volume reduction at month +12 .

Relationship of Progression Free Survival and MRI Tumour Volume

Nine patients have relapsed with a projected 5-year relapse free survival of $80 \%$; of which eight had an isolated local failure and only one relapse had a component of distant failure (Fig. 6). No factors were associated with relapse, specifically initial T1 volume $(p=0.52)$, initial T2Flair volume $(p=0.93)$, pathological subtype $(p=0.12), k i 67 \%(p=0.42)$, presence of enhancement $(p=0.38)$, Berger-Sanai Classification Giant Glioma $(p=0.85)$ or delayed referral for IMRT $(p=0.34)$. Additionally, the extent of T1 or T2Flair volume reduction at month +3 or month +12 was not associated with improved progression free survival.

\section{Functional Outcome}

At month +12 there was an improvement in ECOG compared with baseline with all patients either ECOG 0-1 and the ECOG 0 percentage increased from $40-59 \%$. This was similar in month +36 with $84 \%$ accessible patients being ECOG $0-1$. There was one patient with a reduction in ECOG score in the year post-treatment which normalised in the 3-year follow-up period.

In regards to employment, 22 patients could work prior to RT, with 7 patients having major impairment and 3 patients retired. Of these 22 patients working at baseline, 17 were accessible in their third year post IMRT and all remained employed. Five patients of the 17 patients had moved to higher roles, whilst 3 had residual deficits and were employed at a lower level or with restricted duties.

\section{Discussion}

For tumours of the insular region harbouring an IDH mutation there is the potential for long-term survival especially the subgroup with additional 1p19q co-deletion [1-4]. Initial decision-making requires maximising progression free survival without causing treatment related morbidity that could impact on performance status. This study demonstrates excellent tumour volume reduction and progression free survival at early time points following IMRT and temozolomide in a high-risk group of patients with 
significant residual disease at time of management. Importantly, functional status has been maintained or improved from their pre-treatment level.

Surgical approach in the insular region generally requires a sophisticated neurosurgical approach to minimise the morbidity of resection. The neurosurgical issues that increase intraoperative risk include presence of middle cerebral artery branches (particularly small perforating vessels), involvement of the basal ganglia, adjacent speech connections for the dominant hemisphere and the diffuse nature of these tumours with poor demarcation between tumour and normal brain. Preoperative evaluation may include utilising 2HG spectroscopy to determine the presence of an IDH mutation [24]; functional MRI especially with left sided insular tumours to determine Broca's area [25]; and PET scans to identify regions of higher grade for targeted resection guidance utilising awake craniotomy [26], functional cortical mapping, realtime MRI, endoscopic resection or a combination of these procedures can potentially allow a better understanding of the potential extent of resection $[5,27]$.

The survival benefit of maximal debulking for IDH mutated tumours remains uncertain. Retrospective analyses have attempted to clarify the relationship with residual disease and survival, however interpretation has been difficult given the varied adjuvant therapy policies adopted and the likely differing outcomes from subgroup of AAmut and AOD. The historical data describing a relationship between volume of residual disease and outcome relates to an era prior to molecular classification. The landmark 2008 UCSF research demonstrating the adverse impact of small residual disease volumes on progression-free survival and overall survival predated IDH mutation testing [28]. In this study, there was no uniform policy on role of adjuvant RT, though noting that whilst no patients with complete resection received upfront $\mathrm{RT}$, only $32 \%$ of patients with subtotal resection proceeded to RT. For the total group, RT demonstrated a benefit in progression-free survival, however a negative effect on overall survival, presumably related to those patients receiving RT having worse prognostic features. Of note is that insular tumour location was the only brain regional subsite with a significantly worse progression-free and overall survival compared to frontal lobe tumours. This may reflect the need to consider the insular region as a subspecialised site for management protocols.

The most detailed analysis relating to extent of residual disease as a prognostic factor for tumours with IDH mutation is from Rotterdam published in 2018 [29]. This included 28 patients with insular glioma, and that subsite was associated with a higher volume of residual disease after surgery. This study demonstrated a significant association for overall survival with surgery to minimise residual disease for 112 patients with low grade IDH mutated astrocytoma. This existed for all residual volumes from 0.1-5.0 $\mathrm{cm}^{3}$ to $>15 \mathrm{~cm}^{3}$; though it should be noted that the use of adjuvant radiation therapy was limited to only $49 \%$ of patients. This association of improved survival and extent of residual disease was not demonstrated in patients with oligodendroglioma.

An approach with IMRT and temozolomide which is aimed at minimising postoperative morbidity should be designed to optimise the therapeutic relationship, with progression-free survival and avoidance of late toxicity. Whilst this study is limited in description of late effects of IMRT, the functional endpoints of 
excellent performance status and sustained employment suggest that in the median follow-up period of survivors the impact of IMRT is acceptable. This has also been demonstrated in a larger cohort from multiple brain regional sites where functional status in the years post IMRT is high, and most latent impact arises from the immediate postoperative morbidity [13, 30-32].

As knowledge of IDH mutated glioma expands then the potential to predict more adverse tumour subsites may infer a need for a more aggressive initial approach aimed at near-total resection. An expanding knowledge of molecular features, such as CDKN2A heterogenous deletion [33], may then become factors that influence the utilisation of a more aggressive approach with repeat craniotomy seeking maximal tumour reduction prior to delivery of IMRT.

\section{Conclusion}

Large tumour volume reduction of IDHmut AG involving the insular cortex post-IMRT provides excellent tumour control, post-treatment performance status and survival. There was no significant impact of residual post-surgical tumour volume when IMRT was utilised. This approach should be considered as a favourable alternative to potentially morbid debulking surgery for this patient cohort.

\section{Declarations}

Ethics approval and consent to participate:

Data for this study was stored and retrieved from an Ethics approved prospective database approved by the Northern Sydney Local Health District Human Research Ethics Committee (reference LNR/15/HAWKE355). Consent was obtained via a clearly stated opt out procedure.

Consent for publication:

Not applicable

Availability of data material:

The data generated and analysed during the current study is not publicly available due to patient confidentiality but will be available for sharing after local institutional ethics approval.

Conflicts of interest/competing interests

The authors have no conflicts of interest to declare.

Funding:

This research did not receive any specific grant from funding agencies in the public, commercial or notfor-profit sectors. 
Authors contributions:

All authors contributed to the study concept. Study was designed by MB. Material preparation, data collection and analysis were performed by MB and GM. The first draft of the manuscript was written by $\mathrm{MB}$ and $\mathrm{GM}$. All authors read and approved the final manuscript.

Acknowledgements:

Not applicable

\section{References}

1. Yan H, Parsons DW, Jin G, et al. (2009) IDH1 and IDH2 Mutations in Gliomas. N Engl J Med; 360:765-773. https://doi.org/10.1056/NEJMoa0808710.

2. Cairncross JG, Wang M, Jenkins RB et al. (2014) Benefit from Procarbazine, Lomustine, and Vincristine in Oligodendroglial Tumors is Associated With Mutation of IDH. J Clin Oncol. 32(8):78390. https://doi: 10.1200/JC0.2013.49.3726.

3. Olar A, Wani KM, Alfaro-Munoz KD, et al. (2015) IDH mutation status and role of WHO grade and mitotic index in overall survival in grade II-III diffuse gliomas. Acta Neuropathol. 129(4):585-96. https://doi: 10.1007/s00401-015-1398-z.

4. Metellus P, Coulibaly B, Colin C, et al. (2010) Absence of IDH Mutation Identifies a Novel Radiologic and Molecular Subtype of WHO Grade II Gliomas With dismal Prognosis. Acta Neuropathol. 120(6):719-29. httsp://doi:10.1007/s00401-010-0777-8.

5. Racine CA, Li J, Molinaro AM, Butowski N, Berger MS. (2015) Neurocognitive Function in Newly Diagnosed Low-grade Glioma Patients Undergoing Surgical Resection With Awake Mapping Techniques. Neurosurgery. 77(3):371-9; https://doi:10.1227/NEU.0000000000000779.

6. Duffau H. (2018) Is non-awake surgery for supratentorial adult low-grade glioma treatment still feasible? Neurosurg Rev. 41(1):133-139. https://doi: 10.1007/s10143-017-0918-9.

7. Martino J, Gomez E, Bilbao JL, Dueñas JC, Vázquez-Barquero A. (2013) Cost-utility of maximal safe resection of WHO grade II gliomas within eloquent areas. Acta Neurochir (Wien). 2013 Jan;155(1):41-50. https://doi: 10.1007/s00701-012-1541-8.

8. Hervey-Jumper SL, Berger MS. (2016) Maximizing safe resection of low- and high-grade glioma. J Neurooncol. 130(2):269-282. Epub 2016 May 12. Review. PMID:27174197.

9. Aghi MK, Nahed BV, Sloan AE, Ryken TC, Kalkanis SN, Olson JJ. (2015) The role of surgery in the management of patients with diffuse low grade glioma: A systematic review and evidence-based clinical practice guideline. J Neurooncol. 125(3):503 - 30. Httsp://doi:10.1007/s11060-015-1867-1.

10. Patel T, Bander ED, Venn RA, et al. (2017) The Role of Extent of Resection in IDH1 Wild-Type or Mutant Low-Grade Gliomas. Neurosurgery. 82(6):808-814. doi: 10.1093/neuros/nyx265. 
11. Berger M, Sanai, Polley M. (2010) Insular glioma resection: assessment of patient morbidity, survival and tumour progression. J Neurosurg. 112(1), 1-9. doi: 10.3171/2009.6.JNS0952.

12. Back M, LeMottee M, Crasta $C$, et al. (2017) Reducing radiation dose to normal brain through a risk adapted dose reduction protocol for patients with favourable subtype anaplastic glioma. Radiat Oncol. 12(1):46. https://doi:10.1186/s13014-017-0782-3.

13. Navarria P, Pessina F, Cozzi L, et al. (2016) Can advanced new radiation therapy technologies improve outcome of high grade glioma (HGG) patients? analysis of 3D-conformal radiotherapy (3DCRT) versus volumetric-modulated arc therapy (VMAT) in patients treated with surgery, concomitant and adjuvant chemo-radiotherapy. BMC Cancer. 16:362. Httsp://doi:10.1186/s12885016-2399-6.

14. Grosu AL, Weber WA. (2010) PET for radiation treatment planning of brain tumours. Radiother Oncol. 96(3):325-7. https://doi:10.1016/j.radonc.2010.08.001.

15. Back M, Jayamanne D, Brazier D, Bailey D, Hsaio E, Guo L, Wheeler H. (2019). Tumour volume reduction following PET guided intensity modulated radiation therapy and temozolomide in IDH mutated anaplastic glioma. Journal of Clinical Neuroscience. 59(68-74). https://doi.org/10.1016/j.jocn.2018.11.005.

16. Louis, D. N., Ohgaki, H., Wiestler, O. D., Cavenee, W. K., Burger, P. C., Jouvet, A., Scheithauer, B. W., \& Kleihues, P. (2007). The 2007 WHO classification of tumours of the central nervous system. Acta neuropathologica, 114(2), 97-109. https://doi.org/10.1007/s00401-007-0243-4.

17. Louis DN, Perry A, Reifenberger G, et al. (2016) The 2016 World Health Organization Classification of Tumors of the Central Nervous System: a summary. Acta Neuropathol. 131(6):803-20. https://doi:10.1007/s00401-016-1545-1.

18. Olar A, Wani KM, Alfaro-Munoz KD, et al. (2015) IDH mutation status and role of WHO grade and mitotic index in overall survival in grade II-III diffuse gliomas. Acta Neuropathol. 129(4):585-96. httsp://doi.org/10.1007/s00401-015-1398-z.

19. Berger M, Hervery-Jumper SL et al. (2016) Surgical assessment of the sinula. Part 2: validation of the Berger-Sanai zone classification system for predicting extent of glioma resection. J Neurosurg. 124(2):428-8. doi: 10.3171/2015.4.JNS1521.

20. Van Den Bent MJ, Erridge S, Vogelbaum M, et al. (2016) Results of the interim analysis of the EORTC randomized phase III CATNON trial on concurrent and adjuvant temozolomide in anaplastic glioma without 1p/19q co-deletion: An Intergroup trial. J Clin Oncol 34. https://doi.10.1200/JC0.2016.34.18_suppl.LBA200.

21. Cairncross G, Wang M, Shaw E, et al. (2013) Phase III trial of chemoradiotherapy for anaplastic oligodendroglioma: long-term results of RTOG 9402. J Clin Oncol. 31(3):337-43. https://doi: 10.1200/JC0.2012.43.2674.

22. van den Bent MJ, Brandes AA, Taphoorn MJ, et al. (2013) Adjuvant procarbazine, lomustine, and vincristine chemotherapy in newly diagnosed anaplastic oligodendroglioma: long-term follow-up of 
EORTC brain tumor group study 26951. J Clin Oncol. 31(3):344-50. https://doi: 10.1200/JCO.2012.43.2229.

23. Wen P, Chang SM, Van den Bent MJ, Vogelbaum MA, Macdonald DR, Lee EQ. (2017) Response Assessment in Neuro-Oncology Clinical Trials. J Clin Oncol. 35(21): 2439-2449. https://doi:10.1200/JC0.2017.72.7511.

24. Choi C, Raisanen JM, Ganji SK, et al. (2016) Prospective Longitudinal Analysis of 2-Hydroxyglutarate Magnetic Resonance Spectroscopy Identifies Broad Clinical Utility for the Management of Patients With IDH-Mutant Glioma. J Clin Oncol. 34(33):4030-4039. doi: 10.1200/JC0.2016.67.1222.

25. Zhang B, Chang K, Ramkissoon S, et al. (2017) Multimodal MRI features predict isocitrate dehydrogenase genotype in high-grade gliomas. Neuro Oncol. 19(1):109-117. doi: 10.1093/neuonc/now121.

26. Heinzel A, Stock S, Langen KJ, Müller D. (2012) Cost-effectiveness analysis of amino acid PETguided surgery for supratentorial high-grade gliomas. J Nucl Med. 53(4):552-8. doi: 10.2967/jnumed.111.097352.

27. Castellano A, Cirillo S, Bello L, Riva M, Falini A. (2017) Functional MRI for Surgery of Gliomas. Curr Treat Options Neurol. 19(10):34. https://doi: 10.1007/s11940-017-0469-y.

28. Sanai N and Berger M. (2008) Glioma extent of resection and its impact on patient outcome. Neurosurgery. 62(4):753-764. https:// doi: 10.1227/01.neu.0000318159.21731.cf.

29. Wijnenga MMJ, French PJ, Dubbink HJ, et al. (2018) The impact of surgery in molecularly defined low-grade glioma: an integrated clinical, radiological, and molecular analysis. Neuro Oncol. 20(1):103-112. https://doi:10.1093/neuonc/nox176.

30. Back M, Jayamanne DT, Brazier D, et al. (2019) Influence of molecular classification in anaplastic glioma for determining outcome and future approach to management. J Med Imaging Radiat Oncol. 63(2):272-280. https://doi:10.1111/1754-9485.12850.

31. Klein M, Heimans JJ, Aaronson NK, et al. (2002) Effect of radiotherapy and other treatment-related factors on mid-term to long-term cognitive sequelae in low-grade gliomas: a comparative study. Lancet. 360(9343):1361-8. https://doi: 10.1016/s0140-6736(02)11398-5.

32. Skrap, M, lus T et al. (2012) Low-grade glioma surgery in eloquent areas: volumetric analysis of extent of resection and its impact on overall survival. A single institution experience in 190 patients. J Neurosurg. 117(6):1039-1052. doi: 10.3171/2012.8.JNS12393.

33. Appay R, Dehais C, Maurage CA, et al. (2019) CDKN2A homozygous deletion is a strong adverse prognosis factor in diffuse malignant IDH-mutant gliomas. Neuro Oncol. 21(12):1519-1528. https://doi:10.1093/neuonc/noz124.

\section{Figures}




\section{T2Flair Volume}

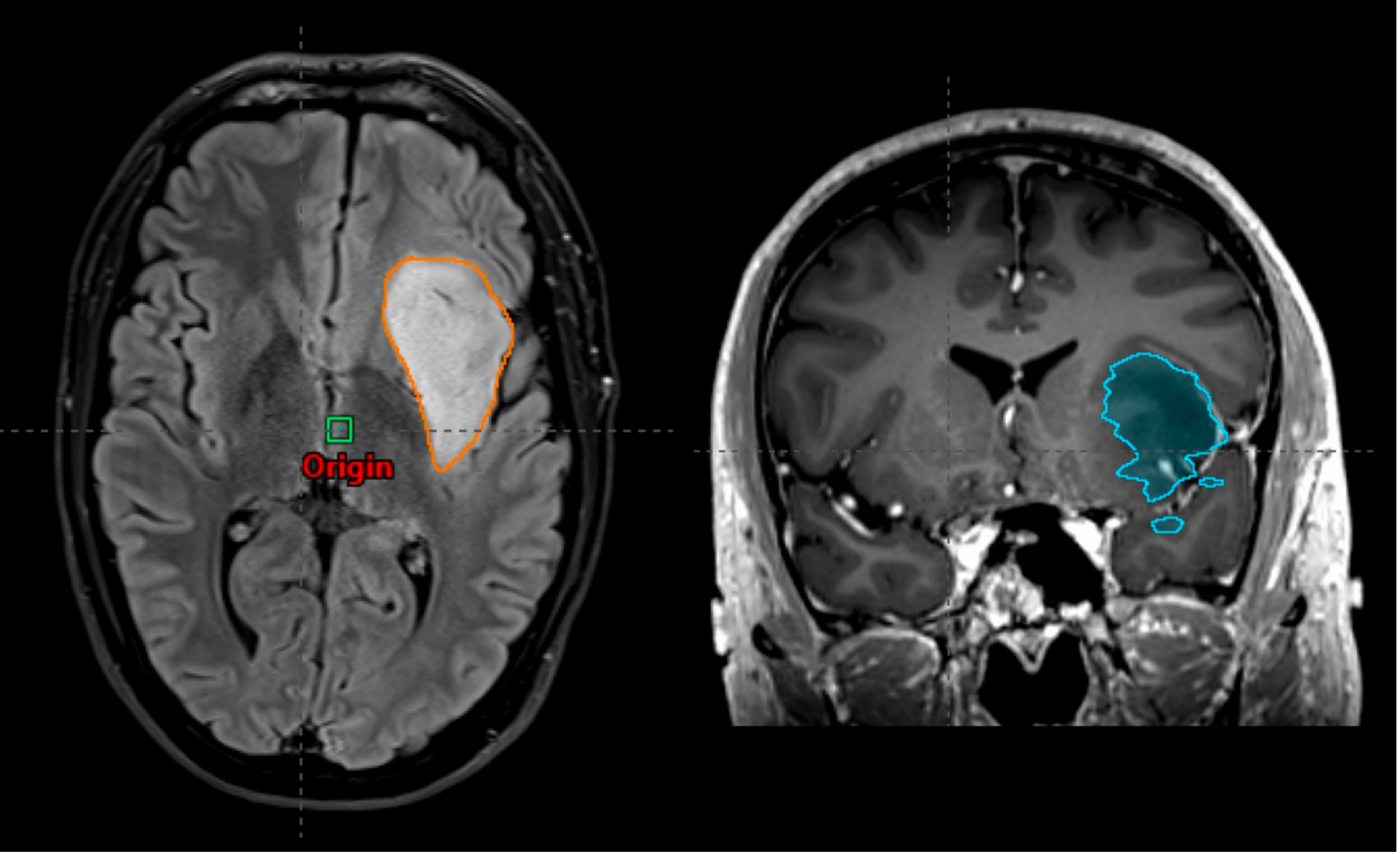

Figure 1

Example of T2Flair and T1 MRI Tumour Volume measurement

T1 Volume 


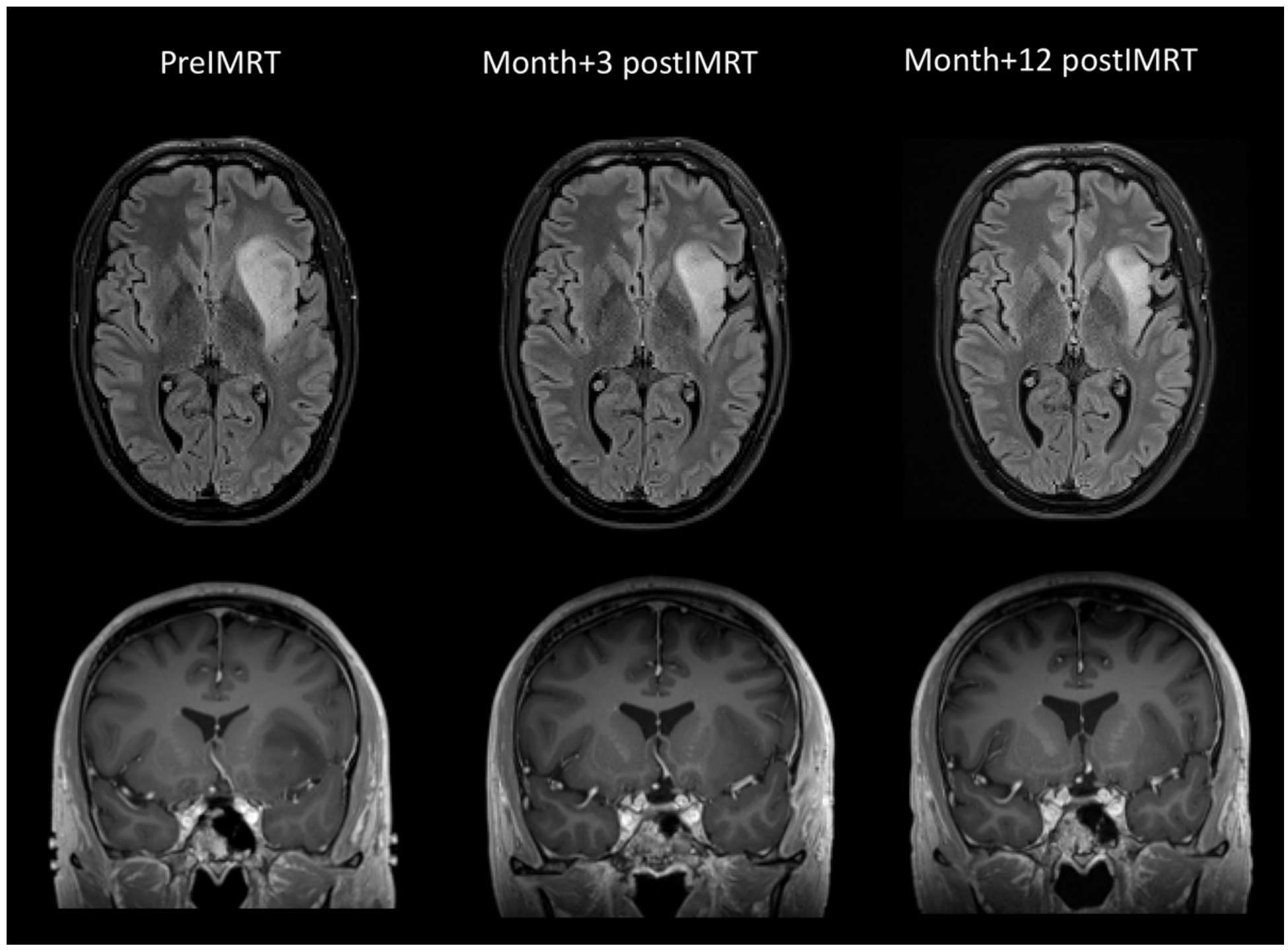

\section{Figure 2}

Example of T2Flair and T1 MRI Tumour Volume assessment over timepoints 

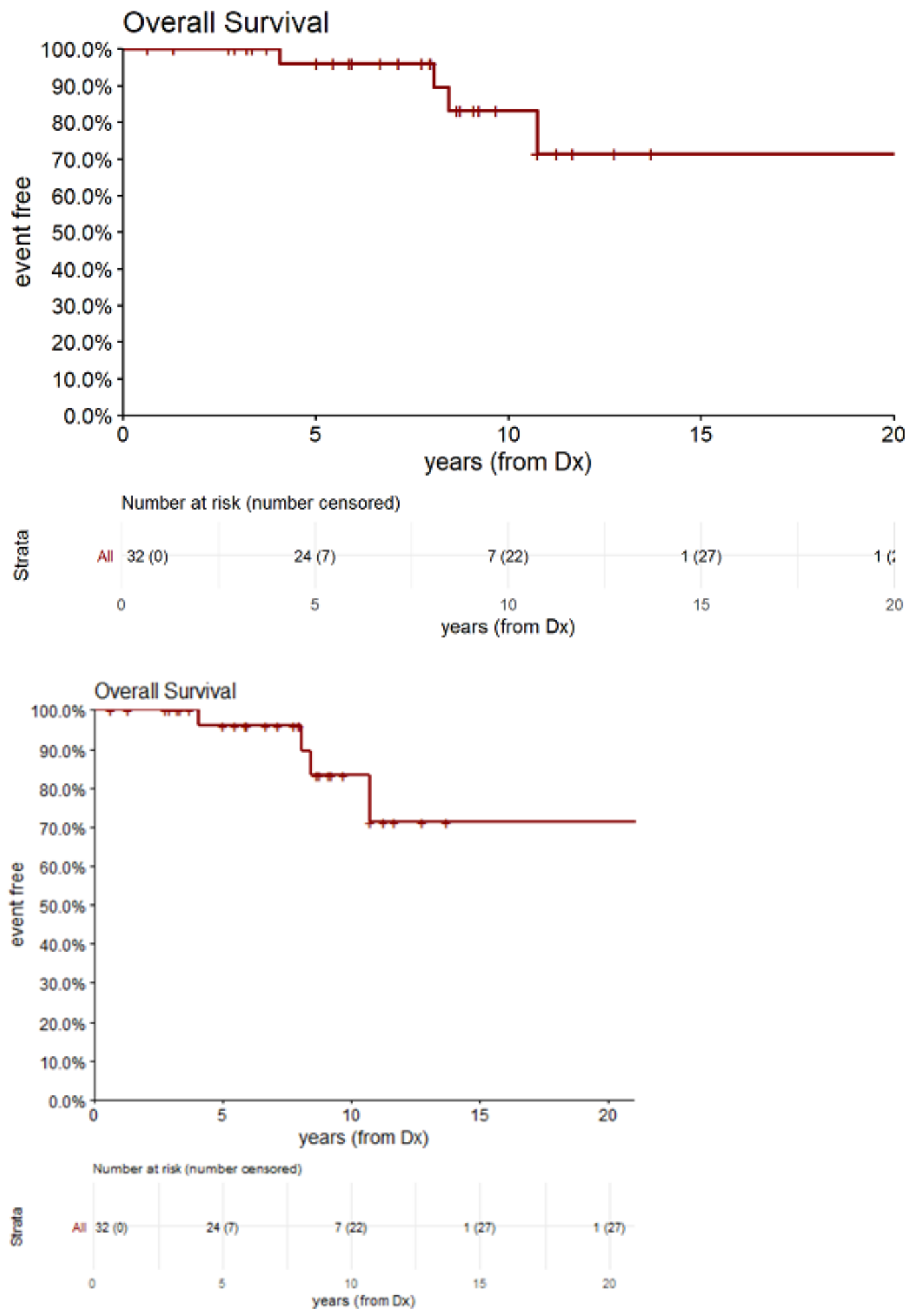

\section{Figure 3}

Overall survival for all patients from start of IMRT $(n=32)$ 

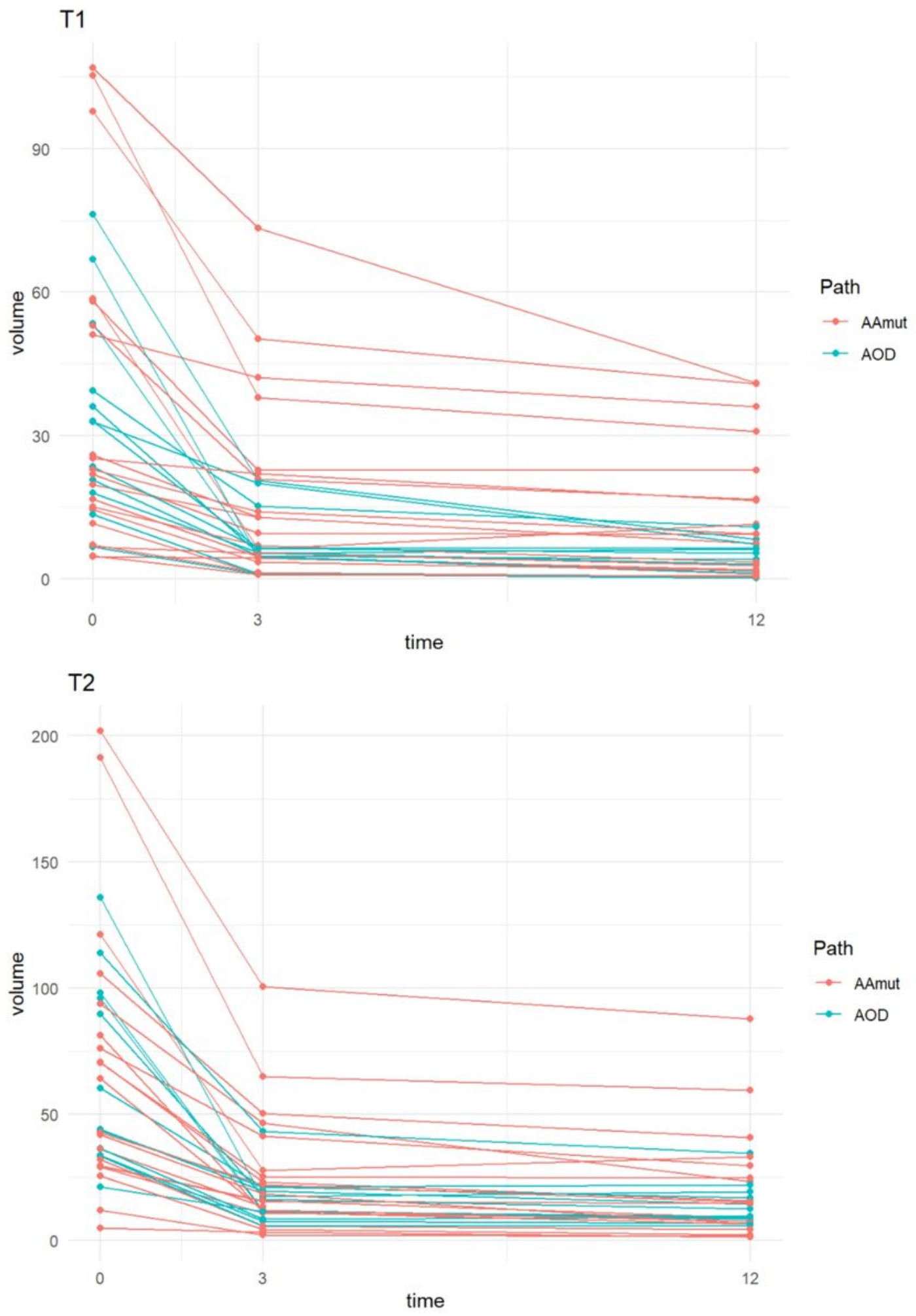

\section{Figure 4}

Individual patient absolute MRI Tumour Volume (T1 and T2Flair) reductions at month+3 and month+12 post-IMRT for pathological subtype. 
Maximum reduction in T1 volume

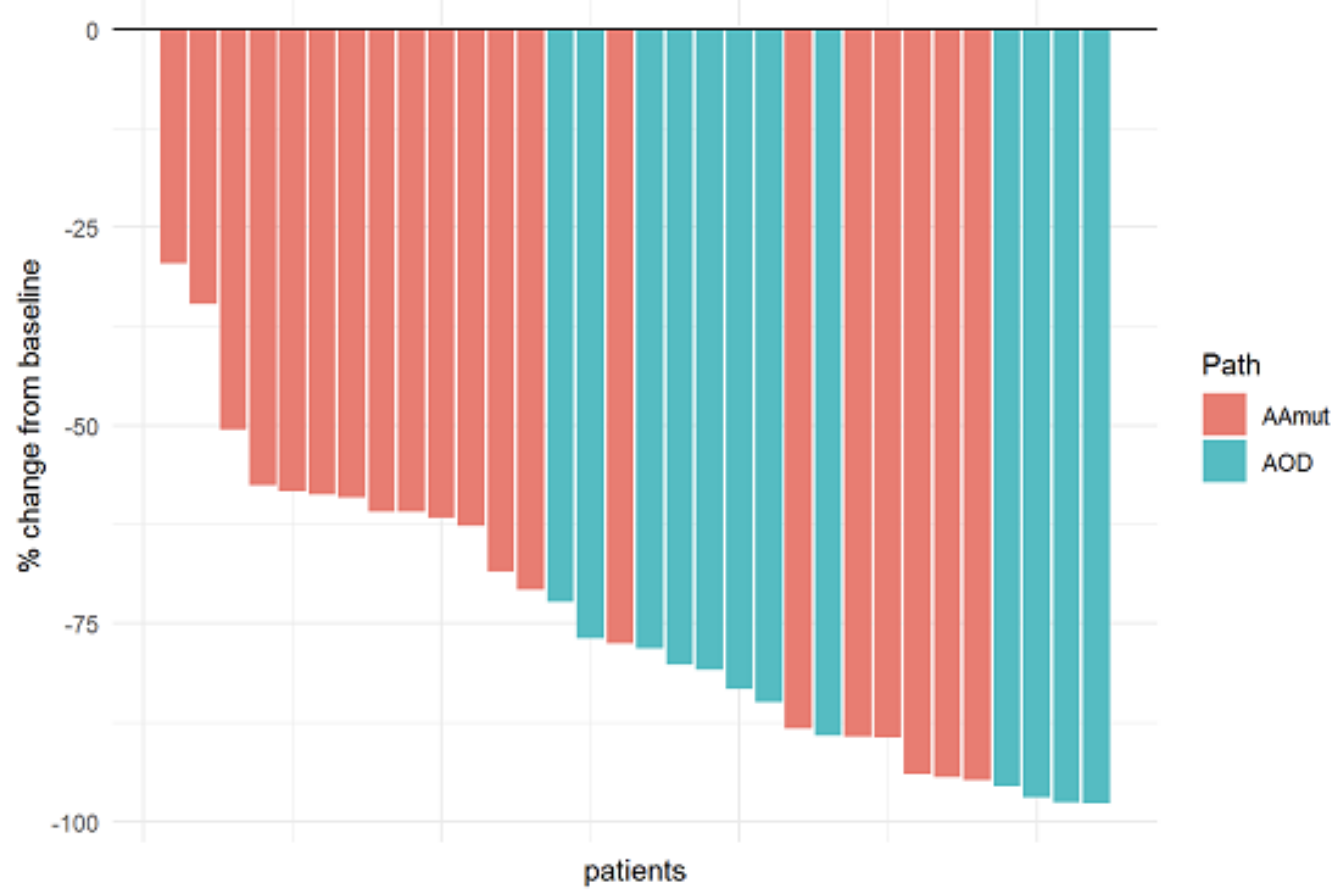

Maximum reduction in $\mathrm{T} 2$ volume

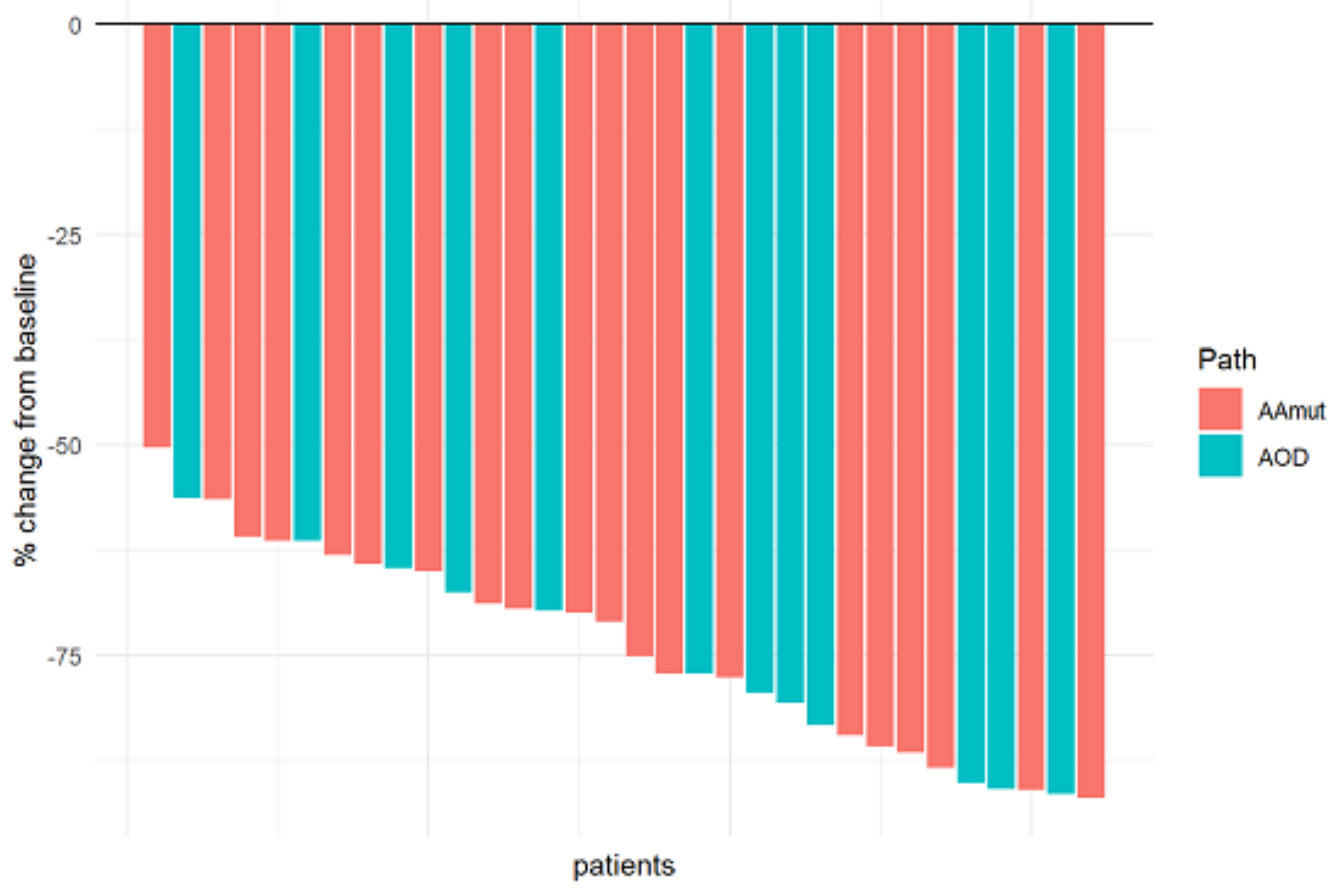

Figure 5

Waterfall plot of individual patient percentage MRI Tumour Volume (T1 and T2Flair) reductions at month+12 post-IMRT from baseline for pathological subtype. 

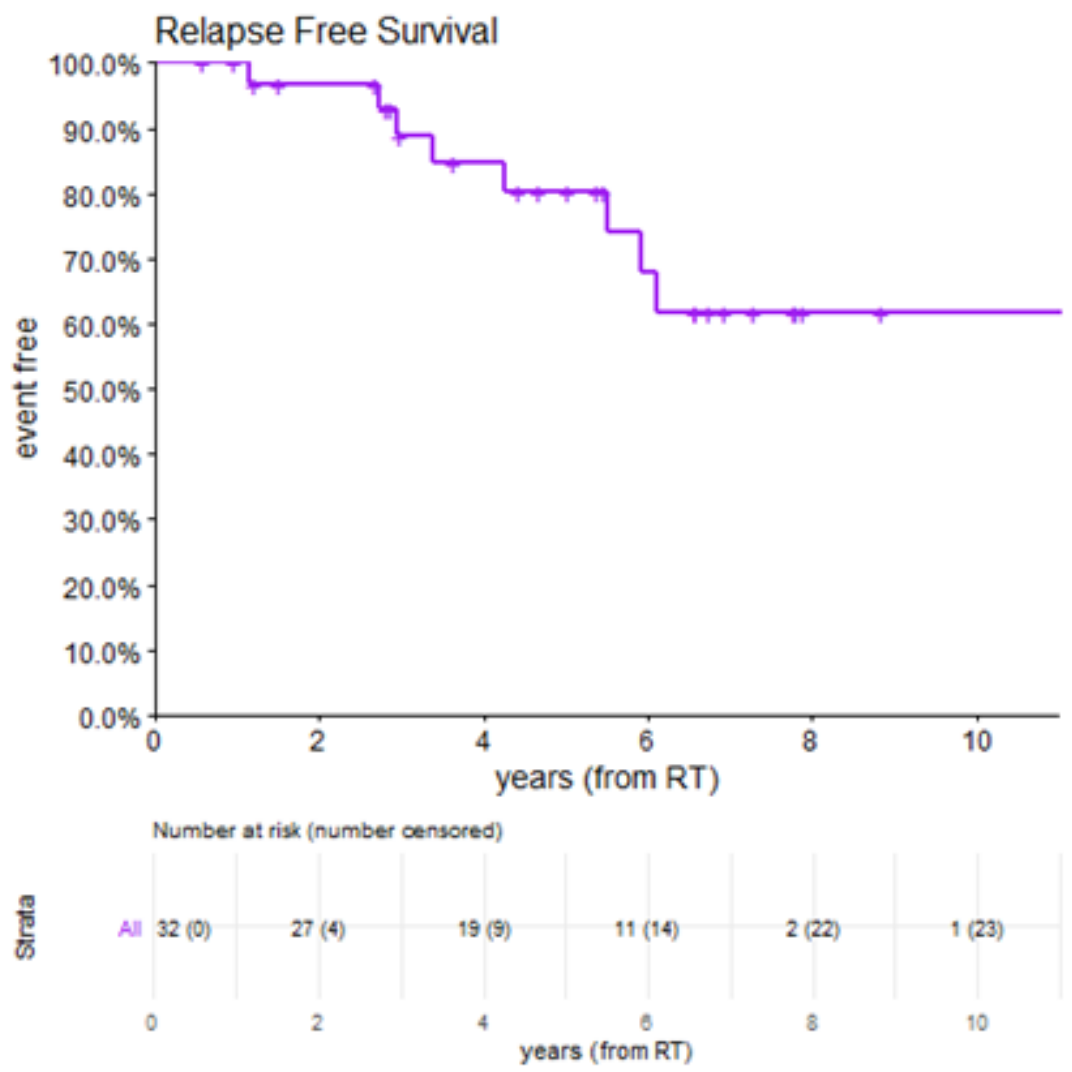

Figure 6

Relapse free survival for all patients from start of IMRT $(n=32)$ 